\title{
Spectrally-invariant behavior of zenith radiance around cloud edges simulated by radiative transfer
}

\author{
J. C. Chiu ${ }^{1}$, A. Marshak ${ }^{2}$, Y. Knyazikhin ${ }^{3}$, and W. J. Wiscombe ${ }^{2,4}$ \\ ${ }^{1}$ Department of Meteorology, University of Reading, Reading, UK \\ ${ }^{2}$ Climate and Radiation Branch, NASA/Goddard Space Flight Center, Greenbelt, MD, USA \\ ${ }^{3}$ Department of Geography, Boston University, Boston, MA, USA \\ ${ }^{4}$ Atmospheric Sciences Division, Brookhaven National Laboratory, New York, NY, USA
}

Received: 28 April 2010 - Published in Atmos. Chem. Phys. Discuss.: 11 June 2010

Revised: 3 November 2010 - Accepted: 19 November 2010 - Published: 30 November 2010

\begin{abstract}
In a previous paper, we discovered a surprising spectrally-invariant relationship in shortwave spectrometer observations taken by the Atmospheric Radiation Measurement (ARM) program. The relationship suggests that the shortwave spectrum near cloud edges can be determined by a linear combination of zenith radiance spectra of the cloudy and clear regions. Here, using radiative transfer simulations, we study the sensitivity of this relationship to the properties of aerosols and clouds, to the underlying surface type, and to the finite field-of-view (FOV) of the spectrometer. Overall, the relationship is mostly sensitive to cloud properties and has little sensitivity to other factors. At visible wavelengths, the relationship primarily depends on cloud optical depth regardless of cloud phase function, thermodynamic phase and drop size. At water-absorbing wavelengths, the slope of the relationship depends primarily on cloud optical depth; the intercept, by contrast, depends primarily on cloud absorbing and scattering properties, suggesting a new retrieval method for cloud drop effective radius. These results suggest that the spectrally-invariant relationship can be used to infer cloud properties near cloud edges even with insufficient or no knowledge about spectral surface albedo and aerosol properties.
\end{abstract}

\section{Introduction}

The transition zone between cloudy and clear air, sometimes called the "cloud halo", is a region of strong aerosolcloud interactions. Based on large eddy simulations, Koren et al. (2009) and Jiang et al. (2009) demonstrated that

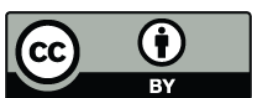

Correspondence to: J. C. Chiu

(c.j.chiu@ reading.ac.uk) a polluted cloud field had smaller cloud halos because cloud drops evaporated faster in polluted air. However, this type of aerosol-cloud interaction is difficult to confirm using traditional data, for two reasons. First, separations between aerosol and cloud using remote sensing techniques are always ambiguous and depend on which variables are being observed and on instrument sensitivities (Charlson et al., 2007; Koren et al., 2008). The transition from cloud to clear could span as little as $50 \mathrm{~m}$ or as much as several hundred meters (Platt and Gambling, 1971; Perry and Hobbs, 1996; Lu et al., 2003; Koren et al., 2007; Su et al., 2008; Redemann et al., 2009). Second, the transition zone is hard to measure because most research aircraft, satellites and ground-based instruments have insufficient spatial and temporal resolution to resolve the transition (Chiu et al., 2009). As a result, aerosol and cloud retrievals near cloud edges are not reliable, considering the combined effects of undetected clouds, 3D cloud radiation, and aerosol property changes (Zhang et al., 2005; Wen et al., 2007; Marshak et al., 2008; Tackett and Di Girolamo, 2009; Twohy et al., 2009; Várnai and Marshak, 2010).

Recently, one-second-resolution zenith radiance measurements from Atmospheric Radiation Measurement program ShortWave Spectrometer (SWS) provide a unique opportunity to analyze the cloud-clear transition zone. Chiu et al. (2009) rigorously defined the transition zone and found a remarkable linear relationship between the sum and difference of radiances at 870 and $1640 \mathrm{~nm}$ wavelengths; this relationship allows us to separate radiative signatures of aerosols and clouds. The intercept of the line depends mainly on aerosol optical depth and size, whereas the slope of the line depends mainly on cloud drop size. Furthermore, this linearity supports the inhomogeneous mixing hypothesis, which predicts that, as a cloud is approached, optical depth increases while the effective drop size remains nearly unchanged. 
Table 1. SBDART input parameters used in the control run and sensitivity tests.

\begin{tabular}{|c|c|c|}
\hline Parameters & Values used in model control run & Values used in sensitivity tests \\
\hline \multicolumn{3}{|l|}{ Spectral } \\
\hline Lower wavelength limit & $0.4 \mu \mathrm{m}$ & \\
\hline Upper wavelength limit & $2.3 \mu \mathrm{m}$ & \\
\hline Spectral resolution & $0.01 \mu \mathrm{m}$ & \\
\hline \multicolumn{3}{|l|}{ Solar } \\
\hline Solar spectrum & MODTRAN_3 & \\
\hline Solar zenith angle & $45^{\circ}$ & \\
\hline \multicolumn{3}{|l|}{ Atmosphere } \\
\hline Atmospheric profile & Mid-latitude summer & \\
\hline Integrated water vapor amount & $3 \mathrm{~cm}$ & \\
\hline Integrated ozone concentration & $0.324 \mathrm{~atm}-\mathrm{cm}$ & \\
\hline \multicolumn{3}{|l|}{ Surface } \\
\hline Surface type & vegetation & black; snow; sand \\
\hline \multicolumn{3}{|l|}{ Cloud } \\
\hline Cloud layer altitude & $1 \mathrm{~km}$ & $5 \mathrm{~km}$ \\
\hline Cloud optical depth at $0.55 \mu \mathrm{m}$ & $0-3$ & \\
\hline Cloud drop effective radius & $4 \mu \mathrm{m}$ & 8 and $16 \mu \mathrm{m}$ \\
\hline Cloud phase function & Henyey-Greenstein & Mie \\
\hline Cloud phase & liquid water & liquid; ice \\
\hline \multicolumn{3}{|l|}{ Aerosol } \\
\hline Aerosol type & rural & urban; oceanic \\
\hline Aerosol optical depth at $0.55 \mu \mathrm{m}$ & 0.2 & 1.0 \\
\hline Aerosol phase function & Henyey-Greenstein & \\
\hline Visibility at $0.55 \mu \mathrm{m}$ & $23 \mathrm{~km}$ & \\
\hline Relative humidity used in the boundary layer aerosol model & $80 \%$ & \\
\hline
\end{tabular}

By extending this work to all wavelengths in the SWS spectra, Marshak et al. (2009) discovered a surprising spectrally-invariant relationship between ratios of zenith radiance spectra in the transition zone. The relationship demonstrates that the shortwave spectra within the transition zone are a linear combination of zenith radiance spectra of purely cloudy and purely clear regions, with a wavelengthindependent weighting function in the transition zone. By invoking the spectral invariance, the high spectral resolution SWS radiance measurements in the transition zone can also be well approximated using only lower spectral resolution measurements.

The main objective of this paper is to confirm the spectrally-invariant behavior found in the SWS observations with radiative transfer calculations. We also study factors that could, in principle, affect the spectrally-invariant behavior. These sensitivity results will provide insights for the improvement of aerosol and cloud property retrievals near cloud edges.

\section{Radiative transfer simulations}

We use SBDART (the Santa Barbara DISORT Atmospheric Radiative Transfer; Ricchiazzi et al., 1998) to compute spectral zenith radiance at wavelengths $400-2300 \mathrm{~nm}$ with $10-\mathrm{nm}$ resolution. Table 1 shows key parameters used in our control run simulation. The extraterrestrial solar spectrum follows the database in MODTRAN at $20 \mathrm{~cm}^{-1}$ spectral resolution. The solar zenith angle is $45^{\circ}$. A standard midlatitude summer atmospheric profile and a vegetation surface albedo are used. The top of the atmospheric grid is $100 \mathrm{~km}$. The total column water vapor amount is set to $3 \mathrm{~cm}$, the total ozone column to $0.324 \mathrm{~atm}-\mathrm{cm}$. Other trace gas amounts are given by default in SBDART.

Cloud bases are located at 1-km altitude with a fixed drop effective radius of $4 \mu \mathrm{m}$. Cloud optical depth varies smoothly across the transition zone. The boundary layer aerosols are characterized using the rural aerosol model in SBDART; the total aerosol optical depth is 0.2 , while the vertical profile of aerosols is determined by setting the visibility parameter 
to $23 \mathrm{~km}$. The spectral aerosol scattering parameters in SBDART depend on humidity; we use $80 \%$ relative humidity. The Henyey-Greenstein phase function is used for both clouds and aerosols.

\subsection{The spectrally-invariant relationship}

The spectrally-invariant behavior found in SWS measurements is derived as follows (Marshak et al., 2009). First, measured zenith radiance $I_{m}$ is normalized by

$I(\lambda ; t)=\frac{\pi \cdot I_{m}(\lambda ; t)}{\mu_{0} \cdot F_{\mathrm{TOA}}(\lambda)}$,

where $I$ is the normalized zenith radiance as a function of wavelength $\lambda$ and time $t ; F_{\mathrm{TOA}}$ is the extraterrestrial solar irradiance; and $\mu_{0}$ is the cosine of solar zenith angle (SZA). By defining variables $y$ and $x$ as:

$y(\lambda ; t)=\frac{I(\lambda ; t)}{I\left(\lambda ; t^{\text {clear }}\right)} ; \quad x(\lambda)=\frac{I\left(\lambda ; t^{\text {cloudy }}\right)}{I\left(\lambda ; t^{\text {clear }}\right)}$,

where $t^{\text {cloudy }}$ and $t^{\text {clear }}$ are times corresponding to fully cloudy and fully clear-sky areas, the spectrally-invariant linear relationship is:

$y(\lambda ; t)=a(t) x(\lambda)+b(t)$,

where $a$ and $b$, the slope and intercept respectively, are a function of $t$ and independent of wavelength $\lambda$. Using the SWS measurements, Marshak et al. (2009) found that Eq. (3) holds for all wavelengths, excluding strong water-vapor absorption bands where divisions by noisy near-zero quantities in Eq. (2) result in large uncertainties.

To test Eq. (3) with radiative transfer simulations, we repeat the same procedure using calculated zenith radiance. For observations, the transition zone is defined by $t^{\text {cloudy }}$ and $t^{\text {clear }}$. For simulations, we use cloud optical depth $\left(\tau_{c}\right)$ to specify the transition zone. We assume that $\tau_{c}^{\text {cloudy }}=3$ in the fully cloudy region, $\tau_{c}^{\text {clear }}=0$ in the fully clear region. Consequently, Eqs. (2) and (3) can be re-written as

$$
\begin{aligned}
& y\left(\lambda ; \tau_{c}\right)=\frac{I\left(\lambda ; \tau_{c}\right)}{I\left(\lambda ; \tau_{c}^{\text {clear }}\right)} ; \quad x(\lambda)=\frac{I\left(\lambda ; \tau_{c}^{\text {cloudy }}\right)}{I\left(\lambda ; \tau_{c}^{\text {clear }}\right)} ; \\
& y\left(\lambda ; \tau_{c}\right)=a\left(\tau_{c}\right) x(\lambda)+b\left(\tau_{c}\right) .
\end{aligned}
$$

We also refer to $a\left(\tau_{c}\right)$ and $b\left(\tau_{c}\right)$ as the slope and the intercept function in the transition zone, respectively. From Eqs. (4) and (5), for the fully cloudy area (i.e., $\tau_{c}=\tau_{c}^{\text {cloudy }}$ ), the slope function is equal to 1 and the intercept function is 0 . For the fully clear area (i.e., $\tau_{c}=\tau_{c}^{\text {clear }}$ ), the slope function is equal to 0 , and the intercept function is equal to 1 .

In next section, we check whether model results support the observed spectrally-invariant behavior (Eq. 5). We also conduct sensitivity tests in Sect. 3 to investigate what factors are dominant in characterizing the slope and intercept functions in the transition zone.
Table 2. Wavelength ranges for bands B1-B5.

\begin{tabular}{lc}
\hline Wavelength range & Band ID \\
\hline $400-870 \mathrm{~nm}$ & B1 \\
$990-1070 \mathrm{~nm}$ & B2 \\
$1180-1290 \mathrm{~nm}$ & B3 \\
$1530-1700 \mathrm{~nm}$ & B4 \\
$2110-2220 \mathrm{~nm}$ & B5 \\
\hline
\end{tabular}

\subsection{Control run simulations}

Similarly to the observed radiance spectra shown in Marshak et al. (2009), our simulations show three main features in Fig. 1a. Firstly, strong water vapor absorption bands occur at wavelengths around 930,1120,1400, and $1900 \mathrm{~nm}$ in simulations. Secondly, due to Rayleigh and aerosol scattering, normalized zenith radiance is higher at shorter wavelengths than at longer wavelengths in the cloud-free condition. Thirdly, in cloudy conditions, normalized zenith radiance at near-infrared wavelengths is close to that at visible wavelengths. Cutting Fig. 1a at a few wavelengths, Fig. $1 \mathrm{~b}$ shows how radiances change their orders in approaching cloudy regions (i.e., increasing cloud optical depth). Near cloudy regions, radiance at $870 \mathrm{~nm}$ exceeds radiances at other wavelengths because the vegetated surface is brighter at $870 \mathrm{~nm}$. In addition, radiance at $2140 \mathrm{~nm}$ is typically lower than that at $1640 \mathrm{~nm}$, due to lower surface reflectance, weaker Rayleigh and aerosol scattering and stronger liquid water absorption.

The ratios $y$ and $x$ calculated from Eq. (4) are plotted in Fig. $1 \mathrm{c}$ for $\tau_{c}$ values of 2,1 , and 0.5. In Fig. 1c, to mimic what we did with observations, we exclude water vapor absorption bands to avoid division by small noisy numbers. Consequently, the remaining wavelength bands are 400-870, 990-1070, 1180-1290, 1530-1700 and 2110-2220 nm, denoted as bands B1-B5 in Fig. 1 and Table 2. Overall, for a given cloud optical depth, points in these bands fall along straight lines. Zooming into each individual band (see examples from bands B1 and B5 in Fig. 1d and e), we see a remarkable linearity that confirms the spectrally-invariant hypothesis. However, bands B1 and B5 have their own slopes and intercepts. In band B1, the sum of the slope and intercept for each line is close to 1 , consistent with the finding reported in Marshak et al. (2009). But in band B5, the sum is no longer equal to 1 due to liquid water absorption.

Finally, we extended the above analysis to more $\tau_{c}$ values. As an example, Fig. 1f shows the slope function in the transition zone for bands B1 and B5. Similarly, we can generate plots of intercept function versus cloud optical depth (figures not shown). To better understand what factors dominate the slope and intercept functions in the transition zone, we now discuss our sensitivity results. 

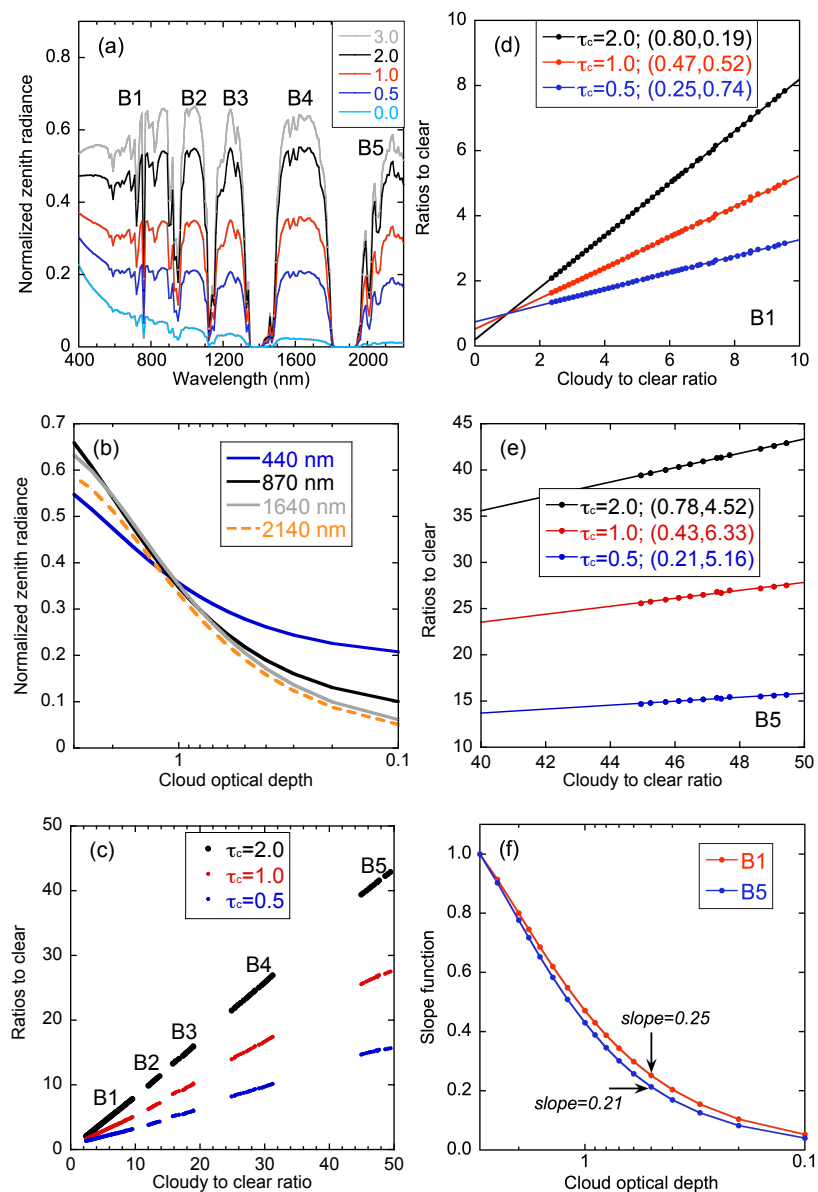

Fig. 1. SBDART simulations from the control run. (a) Simulated zenith radiance at cloud optical depths $\left(\tau_{c}\right)$ ranging from 0 to 3 . Wavelength bands are defined in Table 2. (b) Zenith radiances as a function of cloud optical depth at 440, 870, 1640 and $2140 \mathrm{~nm}$ wavelengths. (c) Plot of radiance ratios to clear ( $y$-variable in Eq. 4) vs. "cloudy to clear ratio" (i.e., $x$-variable in Eq. 4 ) at $\tau_{c}=2.0,1.0$ and 0.5. Values at water vapor absorption bands are excluded in the plot to avoid division smaller numbers. Zoom-in plots at wavelength band B1 and B5 are shown in (d) and (e), respectively. Data points are connected by linear regression lines; the corresponding slope and intercept of the line are listed in parenthesis. By extending the same analyses to more cloud optical depth in the transition zone, a slope function can be derived and is plotted in (f) for bands $\mathrm{B} 1$ and $\mathrm{B} 5$.

\section{Sensitivity tests}

Parameters used in sensitivity tests include cloud and aerosol properties, underlying surface type and the finite field-ofview of a radiometer (as summarized in Table 1). In general, bands B1-B5 have similar sensitivity to all these factors except cloud properties. Therefore, we mainly focus on results in band B1. However, we do discuss both B1 and B5 when the sensitivity parameter is related to cloud properties.
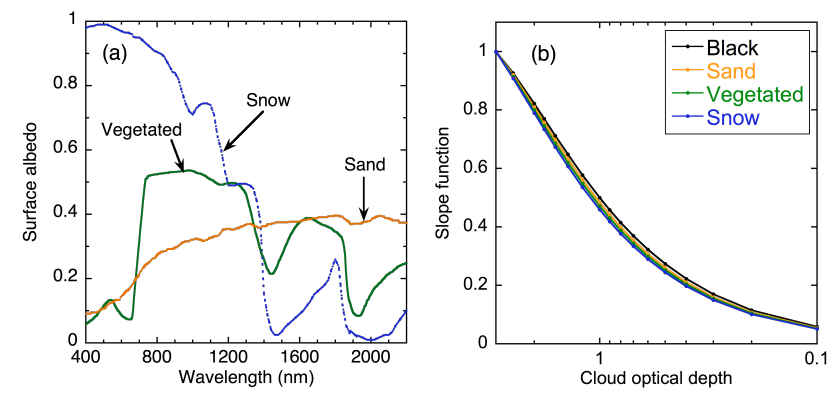

Fig. 2. (a) Spectral surface albedo values used in SBDART simulations for sand, vegetated, and snow surfaces, plotted by orange, green, and blue lines, respectively. (b) Corresponding slope functions, derived from $400-870 \mathrm{~nm}$, depend on cloud otpical depth and underlying surface types.

\subsection{Sensitivity to underlying surface type and aerosol properties}

\subsubsection{Underlying surface type}

We tested four surface types to understand their impacts on the slope function in the transition zone. These types include the black, sand, vegetated and snow surfaces. As shown in Fig. 2a, surface albedo gradually increases with wavelength over sand, whereas surface albedo has a big jump from 0.1 to 0.5 at $700 \mathrm{~nm}$ over vegetations. Among these surface types, the snow surface is the brightest at $400-1200 \mathrm{~nm}$ wavelengths. However, despite such different spectral behaviors in surface albedo, model results indicate that the slope function is not sensitive to the underlying surface type (as shown in Fig. 2b).

\subsubsection{Aerosol properties}

Three aerosol types are used in this sensitivity test: rural, urban and oceanic. Figure 3a-c illustrate spectral dependencies of aerosol optical depth, single scattering albedo and asymmetry factor. In general, oceanic particles have stronger forward scattering and are the least absorbing, while urban particles are the most absorbing. Compared to urban aerosols, rural aerosol particles have a similar size distribution and slightly weaker forward scattering at visible and near-infrared wavelengths. These differences in aerosol optical and microphysical properties, however, have little impact on the slope function (Fig. 3d). Similarly, a change in aerosol optical depth from 0.2 to 1.0 also has negligible impact on the slope function (figures not shown). Overall, the insensitivity of the spectrally-invariant behavior to aerosol properties means that one may still infer cloud properties even with insufficient aerosol information.

Note that in contrast to this paper, Chiu et al. (2009) used normalized zenith radiances at only two wavelengths and plotted them on the sum vs difference plane. Because those 

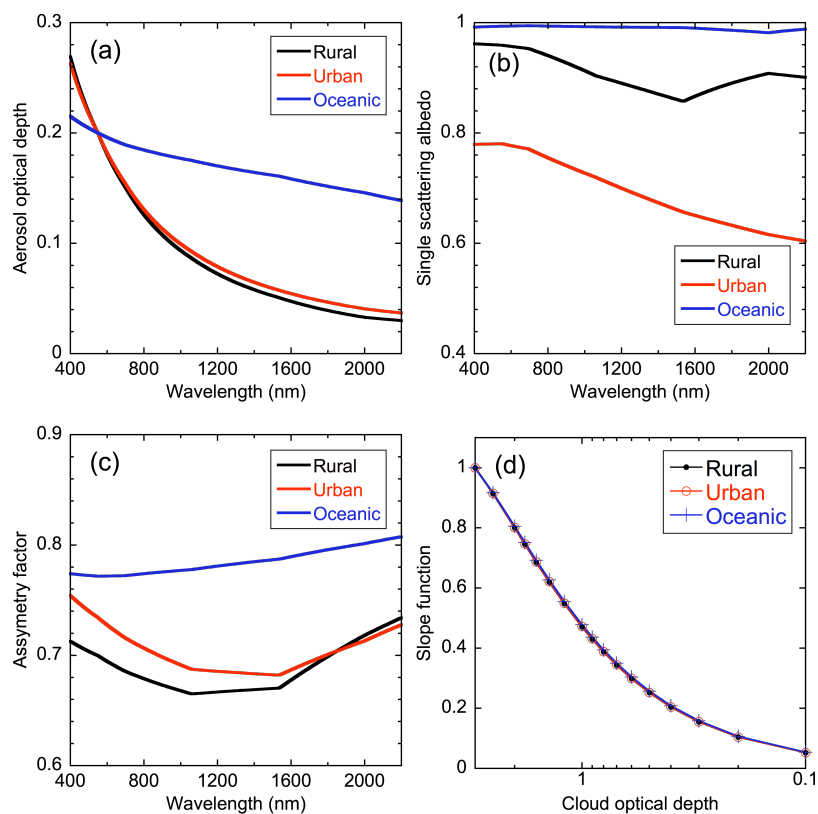

Fig. 3. Optical depth (a), single scattering albedo (b), and assymetry factor (c) for rural (black), urban (red), and oceanic (blue) aerosols, used in the sensitivity test. (d) Corresponding slope functions derived from simulations at $400-870 \mathrm{~nm}$.

radiances were not rescaled by any clear-sky radiances, ratios derived from points on the sum vs difference plane strongly depended on aerosol properties and surface type. On the contrary, a much wider spectral region is taken into account in this paper. All radiances are also rescaled by clear-sky radiances, which in turn reduces the sensitivity to both aerosols and surface type significantly.

\subsection{Sensitivity to cloud properties}

In this section we demonstrate how the slope and intercept of spectrally-invariant relationship are affected by cloud drop effective radius, cloud phase and cloud-base height. Discussions are mainly based on simulations in bands B1 (400$870 \mathrm{~nm}$; not liquid-water absorbing) and B5 (2110-2220 nm; liquid-water absorbing).

\subsubsection{Cloud drop effective radius}

Figure 4 illustrates that the slope and intercept of the spectrally-invariant linear relationship may vary from band to band depending on cloud drop size. With an increasing effective radius from 4 to $8 \mu \mathrm{m}$, data points in band B5 shift toward smaller ratios in both axes, whereas shifts are much less evident in band B1. The dramatic shifts in band B5 are due to a stronger absorption of larger cloud drops; this leads to smaller radiances in numerators of the ratios in Eq. (4). The denominators of the ratios correspond to clear-sky conditions and are independent of drop size. As a result, both

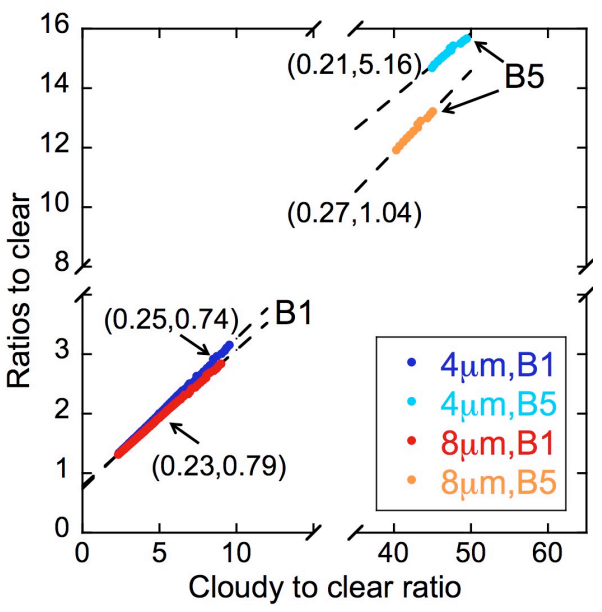

Fig. 4. Spectrally-invariant linear relationships at $\tau_{c}=0.5$, derived from band B1 (400-870 nm) and band B5 (2110-2220 nm), for cloud drop effective radius values of 4 and $8 \mu \mathrm{m}$. Numbers in parentheses are the slope and intercept of the linear regression relationships (dashed lines).

ratios decrease and data points shift toward bottom-left. The small shifts in band B1 can be explained by scattering phase functions; at scattering angle of $45^{\circ}$, the phase function is slightly larger for $4 \mu \mathrm{m}$ than for $8 \mu \mathrm{m}$. The difference in ratios between two cloud drop sizes gradually decreases for shorter wavelengths.

We further extend the above analysis to various cloud optical depths in the transition zone (Fig. 5). Figure 5a and c show that the slope function is weakly sensitive to effective radius in both bands B1 and B5. However, the intercept functions have different behaviors between the two bands (Fig. $5 b$ and d). In band B5, the intercept function strongly depends on effective radius, whereas at $\mathrm{B} 1$ the intercept function does not. Based on these analyses, we conclude:

- As expected, the slope and intercept derived from 400 $870 \mathrm{~nm}$ wavelengths are mainly determined by cloud optical depth and weakly sensitive to cloud drop effective radius

- The slope derived from 2110-2220 nm wavelengths is mainly determined by cloud optical depth; the intercept is mainly determined by cloud drop effective radius

The high sensitivity of the intercept function to cloud drop effective radius at $2110-2220 \mathrm{~nm}$ wavelengths may lead to major application in understanding cloud growth/evaporation processes in the transition zone. A schematic illustration is shown in Fig. 6, using two cloud drop sizes ( 4 and $8 \mu \mathrm{m})$ to characterize the transition zone. For example, we can assume that drop size in the cloudy region is $8 \mu \mathrm{m}$ and remains constant toward the clear region. Another example is that the drop effective radius changes from $8 \mu \mathrm{m}$ in the cloudy region 

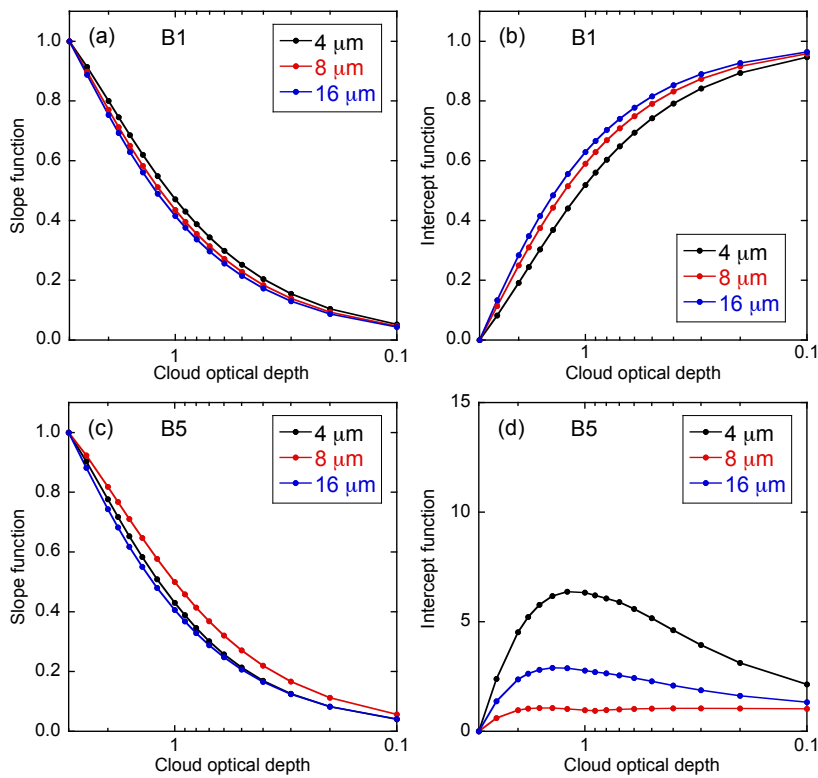

Fig. 5. (a) Slope and (b) intercept functions derived from band B1 (400-870 nm wavelengths). (c) and (d) are the same as (a) and (b), but derived from band B5 (2110-2220 nm wavelengths). Each plot shows results for three cloud drop effective radius values: 4,8 , and $16 \mu \mathrm{m}$.

to $4 \mu \mathrm{m}$ near the clear region. Therefore, with two sizes of 4 and $8 \mu \mathrm{m}$, we have a total of four cases to simulate the cloud drop size change in the transition zone, denoted as cases AD.

Case A presents a bigger cloud drop effective size in cloudy regions and a smaller size towards cloud-free regions, which supports the hypothesis of homogenous mixing, whereby the effective drop size decreases being away from clouds (Mason and Jonas, 1974). In contrast, case B and D (duplicated from Fig. 4) assume a constant $4 \mu \mathrm{m}$ and $8 \mu \mathrm{m}$ effective radius, respectively, supporting the hypothesis of inhomogeneous mixing, whereby the effective drop size remains unchanged as being away from cloud edge (Latham and Reed, 1977; Baker and Latham, 1979; Baker et al., 1980). Case C presents smaller drops in cloudy regions and bigger drops towards cloud-free regions. The change in cloud effective size in this case is exaggerated for illustration clarity and does not occur in reality.

Figure 6 shows that intercepts are substantially different among these cases. Comparison of A to D shows that if the cloud drop size decreases in the transition from cloud to clear, the intercept increases dramatically from 1 to 6 . Similarly, comparison of C to B shows when the cloud drop size increases in moving from cloud to clear, the intercept decreases substantially. This suggests that the intercept at 2110-2220 nm wavelengths contains information on the growth/evaporation tendency of cloud drops, which can be applied to actual observation and provide evidence of homogeneous or inhomogeneous mixing near cloud edges.

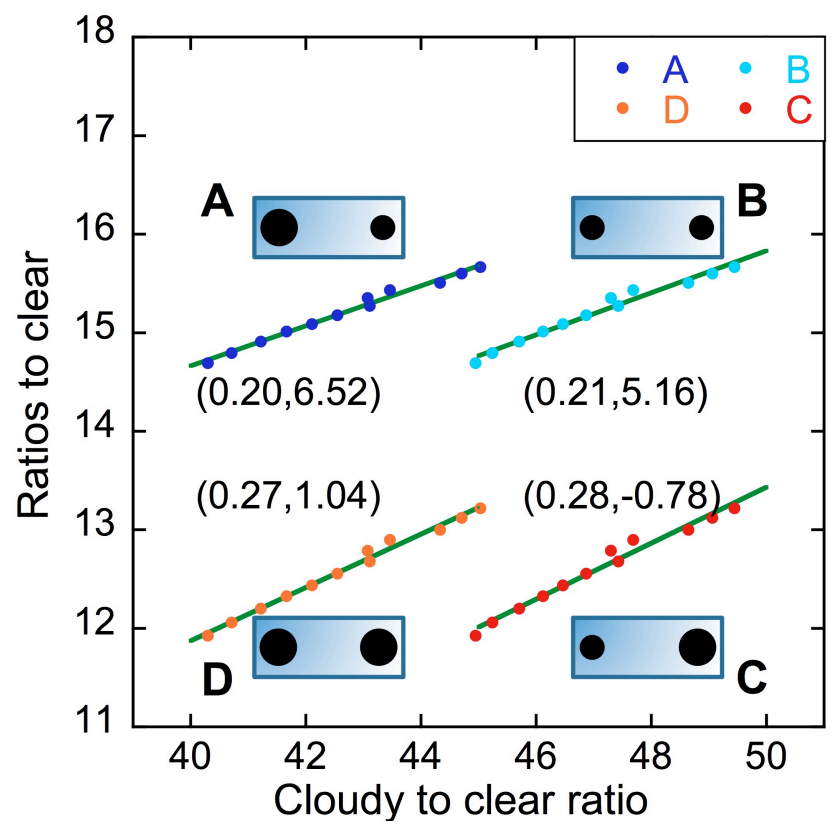

Fig. 6. Spectrally-invariant relationships derived from band B5 $(2110-2220 \mathrm{~nm})$ for situation A-D. Numbers in parentheses are the slope and intercept of the linear relationship (green lines) at $\tau_{c}=0.5$. Each box next to letter A-D schematically illustrates the change of cloud drop effective size in the transition zone. In each box, the filled black circle on the left represents the cloud drop size at $\tau_{c}=3.0$ (the fully cloudy area), while the circle on the right represents the drop size at $\tau_{c}=0.5$ (toward the fully clear area). The smaller circle represents a cloud drop effective radius of $4 \mu \mathrm{m}$, the larger circle represents an effective radius of $8 \mu \mathrm{m}$. Based on these rules, in situation $B$, cloud drop sizes remain a constant of $4 \mu \mathrm{m}$. In situation $\mathrm{D}$, cloud drop sizes remain a constant of $8 \mu \mathrm{m}$. In situation A, cloud drop sizes decrease from $8 \mu \mathrm{m}$ at $\tau_{c}=3.0$ to $4 \mu \mathrm{m}$ toward clearly regions. On the contrary, in situation $\mathrm{C}$, cloud drop sizes increase from 4 to $8 \mu \mathrm{m}$ toward clear regions.

This important finding has not been reported in Marshak et al. (2009). Indeed, data in band B5 were excluded in their analysis because the observed clear-sky radiance in band B5 for their cases was too small to be a divisor. Although data in band B4 were included in their analysis, the difference in single scattering albedo between bands B1 and B4 is less significant than that between bands B1 and B5. As a result, Marshak et al. (2009) did not realize that individual wavelength bands require separate attention and that the intercept in liquid water absorption bands could be used for understanding the growth/evaporation tendency of cloud drops.

\subsubsection{Cloud phase function}

We study sensitivity to cloud phase function by replacing the Henyey-Greenstein phase function with a Mie phase function. Figure 7 shows for band B1, the differences in both slopes and intercepts between these two phase functions are 

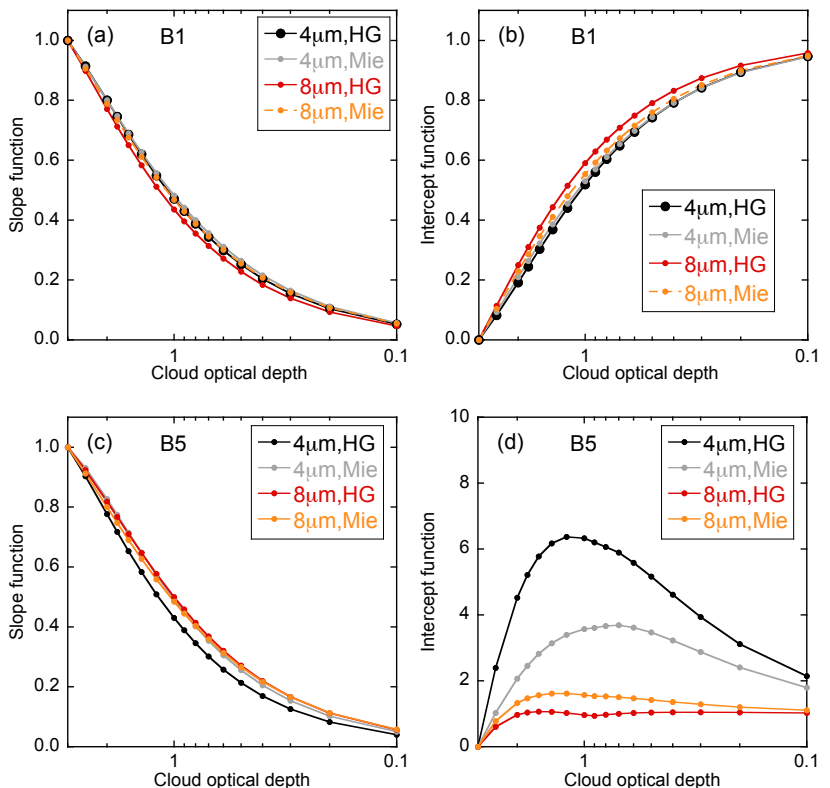

Fig. 7. (a) Slope and (b) intercept functions derived from band B1 (400-870 nm wavelengths). (c) and (d) are the same as (a) and (b), but derived from band B5 (2110-2220 nm wavelengths). Each plot shows results for two cloud drop sizes (4 and $8 \mu \mathrm{m})$ and two types of phase functions (Henyey-Greenstein and Mie).

negligible. For band B5, the slope and intercept are more sensitive to phase function for $4 \mu \mathrm{m}$ of cloud drop size than for $8 \mu \mathrm{m}$. In particular, Fig. 7d shows that with a HenyeyGreenstein phase function, the intercepts for $4 \mu \mathrm{m}$ are generally 2-6 times larger than those for $8 \mu \mathrm{m}$. With a Mie phase function, the difference is reduced to a factor of $2-3$ between 4 and $8 \mu \mathrm{m}$ cloud drop sizes. This reduction implies that, for retrieval purposes, the use of a more realistic phase function is necessary.

\subsubsection{Cloud thermodynamic phase}

This section examines how slope and intercept functions change with cloud thermodynamic phase. In general, ice crystals have stronger forwarding scattering than water drops at $400-2300 \mathrm{~nm}$ wavelengths, except at $\sim 1900 \mathrm{~nm}$. Ice crystals also have stronger absorption than water drops at 2110 $2160 \mathrm{~nm}$, but the opposite is true at $2160-2180 \mathrm{~nm}$. In band B1, liquid- and ice-phase cloud particles have nearly identical absorption properties, and thus cloud phase is expected to have little impact on spectrally-invariant behavior in this band.

Figure 8 confirms that the slope and intercept functions are weakly sensitive to cloud thermodynamic phase in band B1. This insensitivity indicates that if liquid- and ice-phase clouds have the same cloud optical depth variations in the transition zone, both clouds should correspond to similar slope functions regardless of cloud phase. Therefore, cloud optical depth remains the most crucial determinant in the
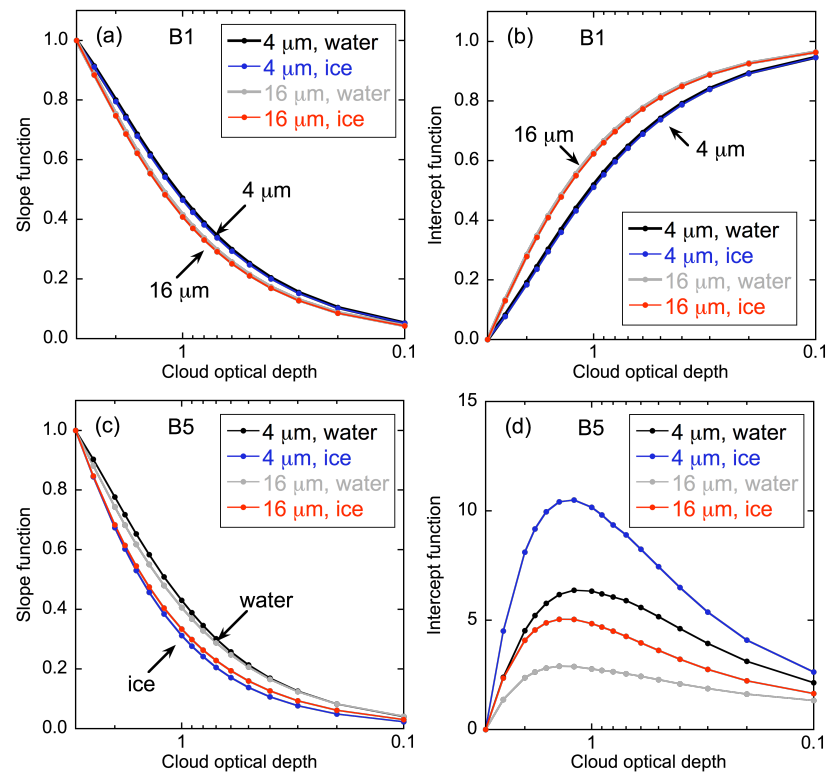

Fig. 8. Same as Fig. 5, but for liquid- and ice-phase clouds with effective sizes of 4 and $16 \mu \mathrm{m}$.

slope function in band B1. Figure 8 also confirms that cloud phase has bigger impact in band B5 than in B1, especially on the intercept function due to the absorption difference between liquid- and ice-phase. The wide spreading curves in Fig. 8d indicate that cloud phase can be checked using the intercept function, especially in the middle of the transition zone where has the largest change in the intercept.

\subsubsection{Finite field-of-view}

This section investigates effects of a finite FOV on the spectrally-invariant behavior in the transition zone. The FOV effect is examined by changing cloud-base height. At a given FOV of $1.4^{\circ}$ (same as the FOV of the ARM shortwave spectrometer), cloud-base height at $1 \mathrm{~km}$ and $5 \mathrm{~km}$ correspond to a FOV of $25 \mathrm{~m}$ and $125 \mathrm{~m}$, respectively. With a nominal $5 \mathrm{~m} \mathrm{~s}^{-1}$ wind speed and 1-s sampling resolution, the $25-\mathrm{m}$ FOV corresponds to 5 sample points, the $125-\mathrm{m}$ corresponds to 25 sample points. We use these sample points in the FOV to calculate average radiance and corresponding spectrallyinvariant relationships.

In this sensitivity test, we assume that cloud optical depth decreases from 3 to 0.1 between locations $100-250 \mathrm{~m}$ (Fig. 9a). Points at distance less than $100 \mathrm{~m}$ are fully cloudy with $\tau_{c}=3$, points at distance greater than $250 \mathrm{~m}$ are fully clear (i.e., $\tau_{c}=0$ ). As we expected, simulations (Fig. 9b) show that the slope function for the cloud at $5 \mathrm{~km}$ altitude is more gradual than that for the cloud at $1 \mathrm{~km}$. If we define the distance $d^{*}$ such that

$a\left(d^{*}\right)=e^{-1}$, 


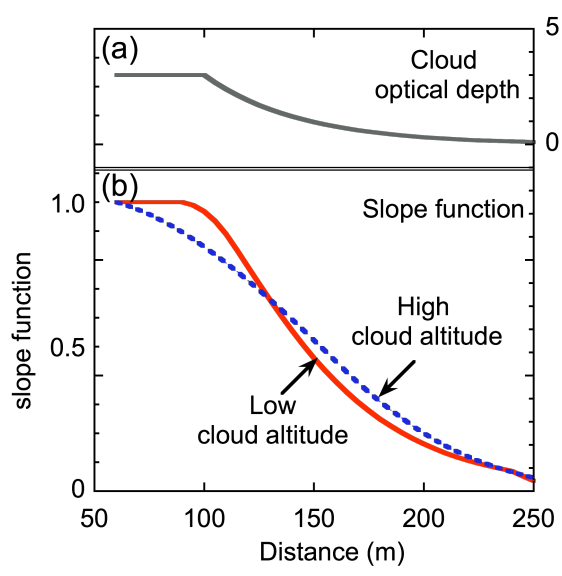

Fig. 9. An ideal case for investigating impact of cloud-base altitude on the slope function of the transition zone. (a) Cloud optical depth as a function of distance. Distance less than $100 \mathrm{~m}$ is in cloudy areas, while distance greater than $250 \mathrm{~m}$ is clear-sky areas. (b) Slope functions for the low and high clouds, as plottted by red-solid line and blue-dashed line, respectively. Slope functions are derived from simulated radiances at $400-870 \mathrm{~nm}$ wavelengths. Results from 2110-2220 nm wavelengths are similar (not shown).

where $a$ is the slope of the spectrally-invariant relationship, we find the resulting $d^{*}$ values are $172 \mathrm{~m}$ and $164 \mathrm{~m}$ for the cloud at $5 \mathrm{~km}$ and $1 \mathrm{~km}$, respectively. This small difference in $d^{*}$ suggests that a finite FOV has a negligible impact on spectrally-invariant behavior in the transition zone.

In the above simulations, we use a fixed wind speed for both low and high clouds; however, wind speed tends to be larger at higher altitudes. Thus, let us consider the following cases. Two clouds are both at $5 \mathrm{~km}$ and have the same relationship between cloud optical depth and distance as Fig. 9a in the transition zone. We further assume that one of these clouds is moving with a nominal wind speed of $5 \mathrm{~m} \mathrm{~s}^{-1}$ and the other is moving with $10 \mathrm{~m} \mathrm{~s}^{-1}$. As a result, the larger wind speed decreases the sampling resolution of radiance, and thus decreases the resolution of the slope function. However, the larger wind speed does not alter the slope function. To summarize, if two clouds have the same cloud optical depth change in the transition zone, cloud base height and the associated FOV effect smooth the slope function, but they only change the $e$-folding distance $d^{*}$ slightly.

\section{Concluding remarks}

We have shown that spectral zenith radiance can be well simulated using radiative transfer calculations with the SBDART model. Ratios of zenith radiance from simulated spectra near cloud edges confirmed spectrally-invariant linear relationships that were observed in ARM shortwave spectrometer measurements. By excluding water vapor absorption bands and separating the remaining wavelengths into five bands, we found that non-absorbing and liquid-water absorbing bands have different linear relationships, and thus require separate attention in sensitivity tests.

We conducted sensitivity tests to better understand what factors dominate in characterizing the slope and intercept functions in the transition zone near cloud edges. These factors include cloud and aerosol properties, underlying surface type, and the finite field-of-view of a radiometer. Overall, the linear relationship at all wavelength bands is insensitive to aerosol properties, underlying surface type, and the finite FOV. Mainly, the spectrally-invariant relationship in the transition zone is sensitive to cloud properties. At nonabsorbing wavelengths (e.g., $400-870 \mathrm{~nm}$ ), the slope and intercept functions are mainly determined by cloud optical depth and are weakly sensitive to cloud drop effective size or phase function. But at liquid-water absorbing wavelengths (e.g., 2110-2220 nm), while cloud optical depth still dominates the slope, cloud absorbing and scattering properties dominate the intercept. This finding could help us understand cloud growth/evaporation processes in the transition zone. For example, we found that the intercept increases dramatically when we simulate a cloud drop size decrease in moving from cloud to clear; if this change in intercept was found in observations, it would tend to support the homogeneous mixing hypothesis.

Furthermore, the high sensitivity of the spectrallyinvariant relationship to cloud properties allows us to better retrieve cloud properties near cloud edges using the slope and intercept functions. These functions are insensitive to assumptions of aerosol properties and surface type, making retrievals using the spectrally-invariant relationships more robust than those retrieved from zenith radiance, which typically require significant prior knowledge about aerosols and spectral surface albedo. Once cloud properties near cloud edges are better understood, the Koren et al. (2009) and Jiang et al. (2009) hypothesis that clouds in more polluted air have sharper edges can be tested with observations.

Although the cloud conditions studied here are strongly inhomogeneous, 3D effects on the spectrally-invariant behavior have not been extensively analyzed yet. However, our preliminary 3D radiative transfer results, presented in the supplement file of this publication, suggest that the spectrally-invariant behavior discovered in SWS measurements is still valid for the 3D simulation world. The slope and intercept functions depend on cloud structure of the transition zone and can be different from their 1D counterparts. More importantly, the general conclusion that "at nonabsorbing bands the slope and intercept functions are mainly determined by cloud optical depth, while at liquid-water absorbing bands cloud optical depth dominates the slope but cloud drop size dominates the intercept" will hold. As a result, changes in intercept in water absorbing bands indicate changes in droplet sizes. 


\section{Supplement related to this article is available online at: http://www.atmos-chem-phys.net/10/11295/2010/ acp-10-11295-2010-supplement.pdf.}

Acknowledgements. This research was supported by the Office of Science (BER, US Department of Energy, Interagency Agreement No. DE-AI02-08ER64562, DE-FG02-08ER64563, DE-FG0208ER54564) as part of the ARM program.

Edited by: B. Mayer

\section{References}

Baker, M. B. and Latham, J.: The evolution of droplet spectra and the rate of production of embryonic raindrops in small cumulus clouds, J. Atmos. Sci., 36, 1612-1615, 1979.

Baker, M. B., Corbin, R. G. and Latham, J.: The influence of entrainment on the evolution of cloud droplet spectra: 1. A model of inhomogeneous mixing. Q. J. Roy. Meteorol. Soc., 106, 581598, 1980.

Charlson, R. J., Ackerman, A. S., Bender, F. A.-M., Anderson, T. L., and Liu, Z.: On the climate forcing consequences of the albedo continuum between cloudy and clear air, Tellus B, 59, 715-727, doi:10.1111/j.1600-0889.2007.00297.x, 2007.

Chiu, J. C., Marshak, A., Knyazikhin, Y., Pilewskie, P., and Wiscombe, W. J.: Physical interpretation of the spectral radiative signatures in the transition zone between cloud-free and cloudy regions, Atmos. Chem. Phys., 9, 1419-1430, doi:10.5194/acp-91419-2009, 2009.

Jiang, H., Feingold, G., and Koren, I.: Effect of aerosol on trade cumulus cloud morphology, J. Geophys. Res., 114, D11209, doi:10.1029/2009JD011750, 2009.

Koren, I., Remer, L. A., Kaufman, Y. J., Rudich, Y., and Martins, J. V.: On the twilight zone between clouds and aerosols, Geophys. Res. Lett., 34, L08805, doi:10.1029/2007GL029253, 2007.

Koren, I., Oreopoulos, L., Feingold, G., Remer, L. A., and Altaratz, O.: How small is a small cloud?, Atmos. Chem. Phys., 8, 38553864, doi:10.5194/acp-8-3855-2008, 2008.

Koren, I., Feingold, G., Jiang, H., and Altaratz, O.: Aerosol effects on the inter-cloud region of a small cumulus cloud field, Geophys. Res. Lett., 36, L14805, doi:10.1029/2009GL037424, 2009.

Latham, J and Reed, R. L.: Laboratory studies of effects of mixing on evolution of cloud droplet spectra, Q. J. Royal Meteo. Soc., 103, 297-306, 1977.

Lu, M.-L., Wang, J., Freedman, A., Jonsson, H. H., Flagan, R. C., McClatchey, R. A., and Seinfeld, J. H.: Analysis of humidity halos around trade wind cumulus clouds, J. Atmos. Sci., 60, 10411059,2003
Marshak, A., Wen, G., Coakley, J., Remer, L., Loeb, N. G., and Cahalan, R. F.: A simple model for the cloud adjacency effect and the apparent bluing of aerosols near clouds, J. Geophys. Res., 113, D14S17, doi:10.1029/2007JD009196, 2008.

Marshak, A., Knyazikhin, Y., Chiu, J. C., and Wiscombe, W. J.: Spectral invariant behavior of zenith radiance around cloud edges observed by ARM SWS, Geophys. Res. Lett., 36, L16802, doi:10.1029/2009GL039366, 2009.

Mason, B. J. and Jonas, P. R.: The evolution of droplet spectra and large droplets by condensation in cumulus clouds, Q. J. Roy. Meteorol. Soc., 100, 23-38, 1974.

Perry, K. D. and Hobbs, P. V.: Influences of isolated cumulus clouds on the humidity of their surroundings, J. Atmos. Sci., 53, 159174, 1996.

Platt, C. M. R. and Gambling, D. J.: Laser radar reflexions and downward infrared flux enhancement near small cumulus clouds, Nature, 232, 182-185, 1971.

Redemann, J., Zhang, Q., Russell, P. B., Livingston, J. M., and Remer, L. A.: Case studies of aerosol remote sensing in the vicinity of clouds, J. Geophys. Res., 114, D06209, doi:10.1029/2008JD010774, 2009.

Ricchiazzi, P., Yang, S. R., Gautier, C., and Sowle, D.: SBDART: A research and teaching software tool for plane-parallel radiative transfer in the Earth's atmosphere, B. Am. Meteorol. Soc., 79, 2101-2114, 1998.

Su, W., Schuster, G. L., Loeb, N. G., Rogers, R. R., Ferrare, R. A., Hostetler, C. A., Hair, J. W., and Obland, M. D.: Aerosol and cloud interaction observed from high spectral resolution lidar data, J. Geophys. Res., 113, D24202, doi:10.1029/2008JD010588, 2008.

Tackett, J. L., and Di Girolamo, L.: Enhanced aerosol backscatter adjacent to tropical trade wind clouds revealed by satellite-based lidar, Geophys. Res. Lett., 36, L14804, doi:10.1029/2009GL039264, 2009.

Twohy, C. H., Coakley Jr., J. A., and Tahnk, W. R.: Effect of changes in relative humidity on aerosol scattering near clouds, J. Geophys. Res., 114, D05205, doi:10.1029/2008JD01099, 2009.

Wen, G., Marshak, A., Cahalan, R. F., Remer, L. A., and Kleidman, R. G.: 3D aerosol-cloud radiative interaction observed in collocated MODIS and ASTER images of cumulus cloud fields, J. Geophys. Res., 112, D13204, doi:10.1029/2006JD008267, 2007.

Várnai, T. and Marshak, A.: Global CALIPSO observations of aerosol changes near clouds, IEEE Remote. Sens. Lett., IEEE Remote. Sens. Lett., doi:10.1109/LGRS.2010.2049982, 2010.

Zhang, J. L., Reid, J. S., and Holben, B. N.: An analysis of potential cloud artifacts in MODIS over ocean aerosol optical thickness products, Geophys. Res. Lett., 32, L15803, doi:10.1029/2005GL023254, 2005. 